\title{
Effect of Lithium Ion Concentration on Thermal Properties in Novel Single-Ion Polymer Electrolyte
}

\author{
By Sang-Woog RYU*
}

Polymer electrolytes consist of heptadecane functionalized poly(ethylene oxide) (PEO) methacrylate and lithium methacrylate were prepared by radical copolymerization and neutralization. The polymer electrolyte was designed to have lithium ion conducting and crystalline domains into an inner-outer double cylinder like array by a macromonomer structure which results in interesting thermal behaviors. In fact, crystalline melting temperature of the heptadecane domain was found at $40^{\circ} \mathrm{C}$ but decreased gradually after introduction of lithium ions into the polymer electrolyte. The more the lithium ion concentration, the lower the melting temperature was observed in DSC study. This result suggests that the crystalline domain size of heptadecane became smaller and broader in their size and size distribution by increasing the lithium ion concentration. The polymer electrolytes reveal $2 \times 10^{-7} \mathrm{~S} \mathrm{~cm}^{-1}$ of room temperature ionic conductivity due to the single-ion nature and quite low content $(\sim 38 \mathrm{wt} \%)$ of the conducting PEO domains. However it was found that there was no active migration of counter anion from the DC-polarization test which indicates a high lithium ion transference number of the polymer electrolyte.

KEY WORDS: Macromonomer / Polymer Synthesis / Polymer Electrolyte / Heptadecane Crystalline / Single-ion / Polarization /

Enhanced safety of portable electronic devices is one of the key concepts in recent lithium ion battery system by replacement of liquid electrolytes with less flammable polymer electrolytes. In addition to the safety issue, it is necessary to obtain several desired properties from a single polymer electrolytes such as a high ionic conductivities, good mechanical properties with a wide electrochemical stability window and high energy density and so on. ${ }^{1,2}$ For that reason, only a limited approach of the polymer electrolyte was reported in terms of several combined but not all of the properties. For example, poly(ethylene oxide) (PEO)-based gel polymer electrolytes exhibit the ionic conductivities as high as $\sim 10^{-3} \mathrm{~S} \mathrm{~cm}^{-1}$ at room temperature but it can not be free from the corrosion or fire hazards arising from the liquid electrolytes. ${ }^{3-5}$ As an alternative, solvent free non-crosslinked solid polymer electrolytes have been suggested and developed for several years but it still remains strong challenge due to the limited segmental motion of the polymer chain, thereby lowering ionic conductivity. ${ }^{6}$

Beside ionic conductivity and mechanical properties, the lithium ion transference number of the electrolyte has recently received a great deal of attention due to the increased demand for further performance gains. In a binary salt system, a gradient in salt concentration change both the lithium diffusion coefficient and the density of charge carriers across the electrolyte and leading to undesired polarization, which eventually reduces the capacity of the battery. ${ }^{7-9}$ Single-ion conductor by tethering the counter anion to the polymer backbone or immobilize in a matrix might be a good strategy to address this problem because the current carried by only mobile lithium cations due to the eliminated polarization. ${ }^{10-17}$
However, lithium ions in such system also become relatively immobile because of the strong ion interactions with tethered anions, leading to low conductivity. ${ }^{18}$ In order to improve the ionic conductivity by enhancing lithium ion mobility, several physical and chemical approaches have been reported. By lowering the ionic interactions between lithium and counter anion, Benrabah et al. reported a lithium sulfornate $\left(\mathrm{SO}_{3} \mathrm{Li}\right)$ single-ion polymer electrolyte with room temperature conductivity of $6 \times 10^{-7} \mathrm{~S} \mathrm{~cm}^{-1},{ }^{19}$ while Fujinami et al. reported a conductivity of $2 \times 10^{-5} \mathrm{~S} \mathrm{~cm}^{-1}$ at ambient temperature with lithium aluminate $\left(\mathrm{AlO}_{2} \mathrm{Li}\right)$-modified compound. ${ }^{11}$ On the other hand, structural control of polymer electrolytes also has been developed by several research groups. For example, recently we have demonstrated that the enhanced ionic conductivity could be achieved by spatial isolation of carboxylate counter anion by self-assembly in a secondary domain of block copolymer electrolytes to promote lithium ion mobility, although the room temperature conductivities for these materials remain in the range of $10^{-6} \mathrm{~S} \mathrm{~cm}^{-1} \cdot{ }^{20}$ Further enhancement in the conductivity through a self-assembled polymer structure, thereby induce decoupled lithium ion motion was reported by Wright and coworkers. ${ }^{21-23}$ Although a large quantity of alkyl chains may hinder the lithium ion conduction, it is not of significance to their system due to the self-assembly between hydrophilic and hydrophobic segment, which results in the formation of an ion conduction channel. Hence, the presence of hydrophobic alkyl chain can be considered to enhance the ionic conductivity if they can be involved in a self-assembly with PEO segment.

The present work explores an amphiphilic single-ion polymer electrolyte that exhibits different thermal behavior 
by a lithium ion concentration. The polymer electrolytes in this study were designed to have a self-assembly not through a block copolymer architecture but derived from an amphiphilic macromonomer structure. Polymer precursors were synthesized by copolymerization of methacrylic acid and amphiphilic methacrylate macromonomer of PEO end-capped with heptadecane crystalline segment. The methacrylic acid function was then transferred to lithium methacrylate by lithiation. Differential scanning calorimetry (DSC) was used to probe thermal transition dependence of the heptadecane crystalline segment on the lithium ion concentration. Electrochemical properties of the prepared polymer electrolytes were characterized in terms of ionic conductivity and DC-polarization.

\section{EXPERIMENTAL}

All the reagents used in this study were purchased from Aldrich and used without further purification otherwise stated. PEO-based amphiphilic macromonomer, (PEO5), end-capped with heptadecane was synthesized as follows. Poly(ethylene glycol) methacrylate $\left[300 \mathrm{~g} \mathrm{~mol}^{-1}, 11.4 \mathrm{~g}\right.$ (38 mmol), $98 \%$, Polysciences. Inc.] was dissolved in anhydrous dimethyl formamide, DMF, $(120 \mathrm{~mL})$ in the presence of triethylamine $(8 \mathrm{~g}, 99 \%)$ and trace amount of hydroquinone $(0.05 \mathrm{~g}, 99 \%)$. After purge with nitrogen for $30 \mathrm{~min}$, octadecanoyl chloride [303 $\mathrm{g} \mathrm{mol}^{-1}, 12 \mathrm{~g} \mathrm{(40} \mathrm{mmol),} \mathrm{99 \% ]} \mathrm{was} \mathrm{slowly} \mathrm{added} \mathrm{and}$ allowed to stir for overnight at room temperature. The yellowish organic layer was then collected by filteration and passed through a silica column using THF as an eluent solvent. The colorless organic liquid was obtained after solvent evaporation [572 $\mathrm{g} \mathrm{mol}^{-1}, 14 \mathrm{~g}(24 \mathrm{mmol}), 63 \%$ of yield]. The synthesized macromonomer, PEO5 $\left(572 \mathrm{~g} \mathrm{~mol}^{-1}, 2 \mathrm{~g}\right.$, $3.5 \mathrm{mmol})$ and methacrylic acid $\left(86 \mathrm{~g} \mathrm{~mol}^{-1}, 0.04 \mathrm{~g}, 0.5 \mathrm{mmol}\right.$, $98 \%$ ) were dissolved in toluene $(10 \mathrm{~mL}$, J.T.Baker) and purged with nitrogen for $20 \mathrm{~min}$. After heating up to $90{ }^{\circ} \mathrm{C}$, azobisisobutyronitrile, AIBN $(0.005 \mathrm{~g}, 0.03 \mathrm{mmol}, 98 \%)$ was added at once and stirred for $24 \mathrm{~h}$. The reaction mixture was then poured into methanol and purified by precipitation from its THF solution into methanol several times. Colorless polymer $(1.6 \mathrm{~g}$, $78 \%$ yield) was obtained after vacuum dried at $100{ }^{\circ} \mathrm{C}$ for overnight. The composition of the resulting polymer was calculated by the integral ratio between macromonomer and methacrylic acid from ${ }^{1} \mathrm{H}$ NMR spectroscopy corresponding 4.0-4.3 ppm $\left(\mathrm{COOCH}_{2}\right.$ of macromonomer) and 1.5-2.0 ppm (backbone proton of methacrylic acid), respectively. The polymer was then dissolved in THF and a quantitative amount of lithium methoxide was added slowly to the solution at room temperature. After a $\mathrm{pH}$ of 7 was reached, the solvent was evaporated and polymer was dried in a vacuum oven at $100{ }^{\circ} \mathrm{C}$ for overnight. The dried polymer was then dissolved in benzene and cast onto stainless steel electrode (diameter: $15 \mathrm{~mm}$ ) and dried in a vacuum oven at $110^{\circ} \mathrm{C}$ for an overnight to give the thickness of 100-300 $\mu \mathrm{m}$. DSC measurements were carried out on a TA Instruments Q100 at a heating rate of $20^{\circ} \mathrm{C} \mathrm{min}^{-1}$. Conductivities of the polymer electrolytes were measured by an impedance spectroscopy using a Solartron 14552 impedance gain/phase analyzer (Solartron Analytical, England) over a frequency range of $1 \mathrm{~Hz}$ to $10^{6} \mathrm{~Hz}$ at $10 \mathrm{mV}$. The electrolyte film was sandwiched between two $15 \mathrm{~mm}$ stainless steel electrodes, which were then assembled into a coin-cell inside argon filled glove box. Lithium symmetric cells fitted with the polymer electrolytes $\left(100-200 \mu \mathrm{m}\right.$ thick, $\sim 1.88 \mathrm{~cm}^{2}$ area) were used to test a polarization behavior under a constant voltage.

\section{RESULTS AND DISCUSSION}

\section{Synthesis of Polymer Electrolytes}

The PEO5 macromonomer having methacrylate functional group was synthesized from coupling poly(ethylene glycol) methacrylate and octadecanoyl chloride as shown in Scheme 1.

The ${ }^{1} \mathrm{H}$ NMR spectrum in Figure 1 shows each characteristic peak of coupled product with reasonable integral ratio. The coupling efficiency of poly(ethylene glycol) methacrylate and octadecanoyl chloride was determined as $96 \%$ from ${ }^{1} \mathrm{H}$ NMR spectroscopic analysis based on the integral ratio at 5.5 (or 6.1) ppm and $0.8 \mathrm{ppm}$ corresponding to double bond and alkyl chain end protons, respectively. It is noticeable that the presence of THF and DMF solvent which are remained after synthetic or purification step has no effect on the polymerization. The GPC diagram in Figure 2 shows that the PEO5 possess symmetrical unimodal peak with narrow molecular weight distribution. It should be noticed that the complicated trace above $35 \mathrm{~mL}$ of elution volume can be ascribed to solvent fluctuation and usually observed in GPC measurement. Thus, once again this coupling reaction appeared to proceed well with high yields.

Number-average molecular weight of PEO5 was determined to $650 \mathrm{~g} \mathrm{~mol}^{-1}$ based on a polystyrene standard and agreed quite well with that of calculated value $\left(572 \mathrm{~g} \mathrm{~mol}^{-1}\right)$. Free radical copolymerization of PEO5 and methacrylic acid was then carried out in toluene with AIBN initiator at $90^{\circ} \mathrm{C}$ as shown in Scheme 1. It has been observed that viscosity of the reaction mixture was gradually increased by the reaction time which suggests the progress of polymerization and corresponding increase of molecular weight. The monomer conversions were around $90 \%$ based on the gravimetric analysis after precipitation and purification steps. Although it is difficult to prepare well-defined polymer structure from a free radical polymerization, the composition of resulting copolymer can be controlled by varying the feed ratio of two monomers. As a result, we have synthesized several precursor copolymers which have a different composition ratio between PEO5 and methacrylic acid based on ${ }^{1} \mathrm{H}$ NMR analysis as shown in Figure 3.

The molecular weights of the copolymer precursors were ranged from $40-92 \mathrm{~kg} \mathrm{~mol}^{-1}$, with around 2.5 of polydispersity index. The single-ion polymer electrolytes (SPEs) were then prepared by the neutralization of methacrylic acid of obtained precursor copolymer with lithium methoxide and the molecular characteristic information is summarized in Table I. 
<smiles>C=C(C)C(=O)OCCOS</smiles><smiles>[3H]C([3H])(C)CC(C)C(=O)OCCOC(=O)C(=C)C</smiles>

PEO5<smiles>C=C(C)C(=O)O</smiles>

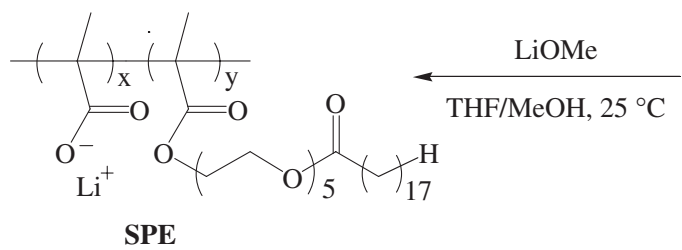<smiles>CC(C)(C)C(=O)OCCOC(=O)C(C)(C)C(=O)OCCOC(=O)C(C)(C(=O)O)C(C)(C)C</smiles>

Precursor

Scheme 1.

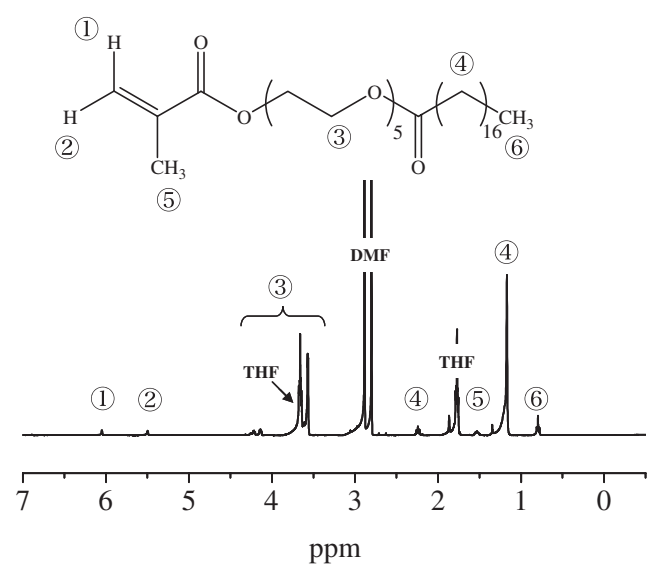

Figure 1. ${ }^{1} \mathrm{H}$ NMR spectrum of PEO5 macromonomer in $\mathrm{CDCl}_{3}$.

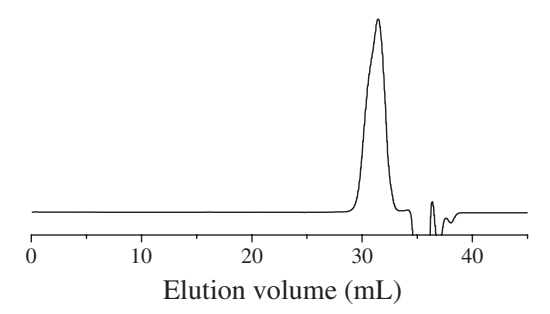

Figure 2. GPC trace of PEO5 macromonomer in $\mathrm{THF}$ at $40^{\circ} \mathrm{C}$.

It should be noted that the weight fraction of ethylene oxide unit is $0.36-0.38$ and these values indicated the major component in matrix are alkyl or backbone domain. The [EO]:[Li] ratio was calculated from the composition between PEO5 which has 5 ethylene oxide units in one molecule and lithium methacrylate. The SPE samples referred to here are based on these numbers.

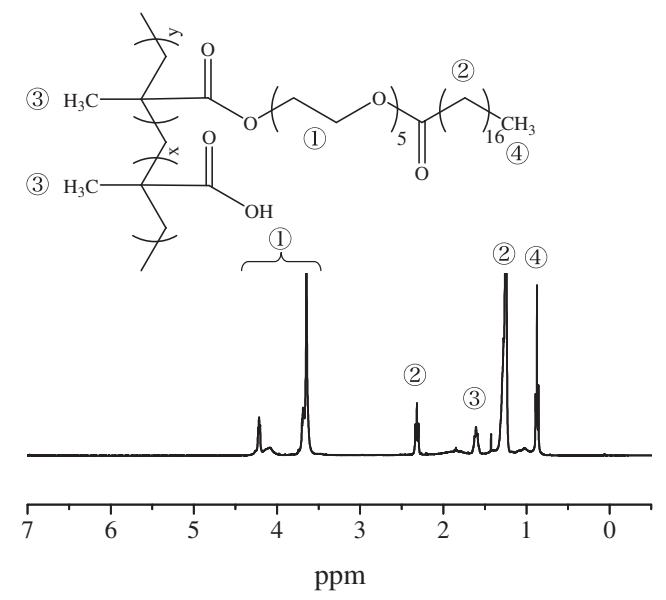

Figure 3. ${ }^{1} \mathrm{H}$ NMR spectrum of polymer precursor in $\mathrm{CDCl}_{3}$.

Table I. Molecular characteristics of single-ion polymer electrolytes

\begin{tabular}{cccccc}
\hline & \multicolumn{3}{c}{ Composition $($ wt \%) } & & Molecular \\
\cline { 2 - 4 } & $\begin{array}{c}\text { Ethylene } \\
\text { oxide unit }\end{array}$ & $\begin{array}{c}\text { Lithium } \\
\text { methacrylate }\end{array}$ & $\begin{array}{c}\text { Alkyl and } \\
\text { backbone }\end{array}$ & [EO]:[Li] & $\begin{array}{c}\text { weight } \\
\left(\mathrm{kg} \mathrm{mol}^{-1}\right)^{\mathrm{b}}\end{array}$ \\
\hline SPE28 & 37.9 & 2.8 & 59.3 & $28: 1$ & 75 \\
SPE23 & 37.7 & 3.4 & 58.9 & $23: 1$ & 92 \\
SPE17 & 37.2 & 4.6 & 58.2 & $17: 1$ & 40 \\
SPE10 & 36.0 & 7.6 & 56.4 & $10: 1$ & 70 \\
\hline
\end{tabular}

${ }^{\mathrm{a}}$ Composition of each polymer was calculated by ${ }^{1} \mathrm{H}$ NMR analysis ${ }^{\text {b}}$ Determined relative to polystyrene in THF at $40^{\circ} \mathrm{C}$.

\section{Thermal Behavior}

The thermal behaviors of the polymer electrolytes were characterized by DSC. Figure 4 shows a melting temperature variation of heptadecane side chain by lithiation in the SPE17 sample.

As seen for the precursor polymer, the sharp endothermic peak of heptadecane side chain was observed at $40^{\circ} \mathrm{C}$ with 


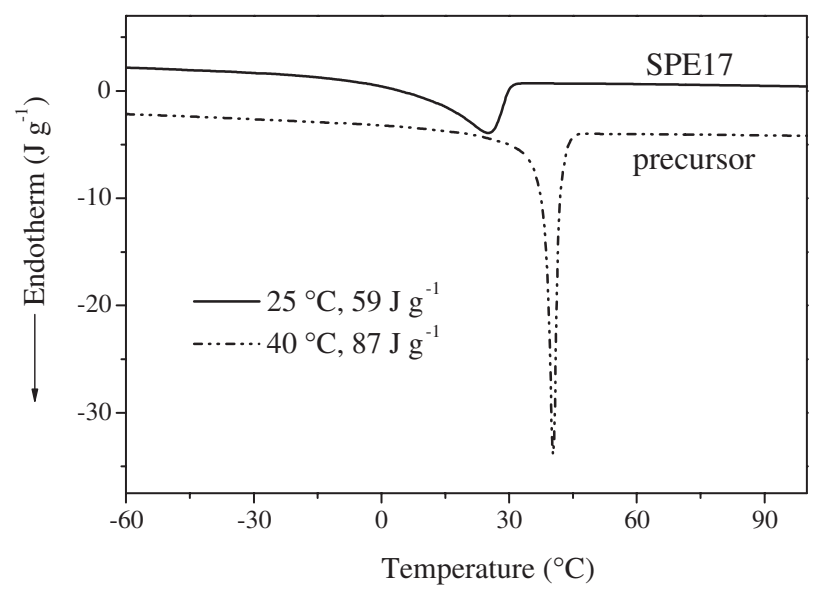

Figure 4. Dependence of heptadecane melting temperature on lithiation in polymer electrolyte system.

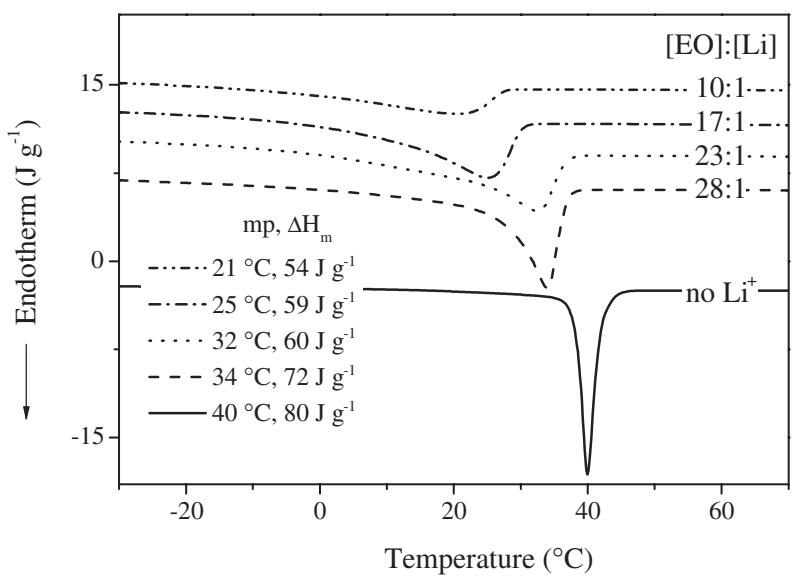

Figure 5. Melting temperature fluctuation of heptadecane crystalline upon lithium ion concentration.

enthalpy of melting $\left(\Delta \mathrm{H}_{\mathrm{m}}\right)$ value of $87 \mathrm{~J} \mathrm{~g}^{-1}$ in the presence of the methacrylic acid counterpart. This peak was, however, shifted to $25^{\circ} \mathrm{C}$ with significant decrease of $\Delta \mathrm{H}_{\mathrm{m}}$ to $59 \mathrm{~J} \mathrm{~g}^{-1}$ upon the lithiation process, which convert the methacrylic acid to corresponding lithium methacrylate. This result strongly suggests that the crystallinity and/or degree of orientation of heptadecane side group were influenced strongly by presence of the lithium ion, which can be coordinated with ethylene oxide unit. Broaden thermal transition can also be explained by the broad size distribution of heptadecane crystalline due to the coordination process. To verify this concept, the melting temperature of the entire polymer was investigated as a function of lithium methacrylate concentration and plotted in Figure 5.

In fact, there was a gradual decrease of melting point and $\Delta \mathrm{H}_{\mathrm{m}}$ from $34{ }^{\circ} \mathrm{C}$ to $21^{\circ} \mathrm{C}$ and $72 \mathrm{~J} \mathrm{~g}^{-1}$ to $54 \mathrm{~J} \mathrm{~g}^{-1}$, respectively, with increasing the lithium concentration from $28: 1$ to $10: 1$, due to the enlarged coordination between lithium ion and ethylene oxide. The more broaden endothermic peaks were also observed with the higher lithium ion concentration, which indicates decreased domain size of the heptadecane crystalline.
Nevertheless, the crystalline melting temperature of the precursor polymer was observed exactly at $40^{\circ} \mathrm{C}$ regardless of the concentration of methacrylic acid. A representative sample is listed in Figure 5 with the name of "no $\mathrm{Li}^{+}$." At this point, one might expect that there is a possible contribution of the methacrylic acid (or methacrylate anion after lithiation process) on the melting temperature of the heptadecane side group because they are distributed along the backbone chain, thereby reduce a packing density. The next step, we have synthesized poly(PEO5) homopolymer and the thermal behavior was investigated by DSC again. Interestingly, we have observed the exact same phase transition at $40{ }^{\circ} \mathrm{C}$ which is corresponding to the heptadecane alkyl chain in the poly(PEO5) homopolymer sample. Therefore the domain size of heptadecane crystalline might not be changed by the presence of methacrylic acid up to 33 mole $\%$ but governed by presence of the lithium ion instead. The physical properties, on the other hand, of the polymer electrolytes seem to be effected by the amount of lithium methacrylate. For example, the poly(PEO5) homopolymer which is in a waxy state at room temperature turned to rubbery with $3.4 \mathrm{wt} \%$ of lithium methacrylate (SPE23).

\section{Electrochemical Properties}

Although the crystalline domain in the polymer electrolyte may decrease the segmental motion of the polymer chain, thereby low ionic conductivity is usually expected, the decoupled lithium ion motion can also be expected if they have a separated conduction channel, coinciding with the Wright's work. ${ }^{21}$ Temperature dependence of the ionic conductivity was measured by the impedance spectroscopy. As a representative sample, SPE23 having EO:Li ratio of 23:1 was heated from 25 to $110^{\circ} \mathrm{C}$ and the conductivities were plotted as a function of temperature.

As shown in Figure 6, there was a steady increase of conductivity upon a heating and achieved $1.8 \times 10^{-6} \mathrm{~S} \mathrm{~cm}^{-1}$ at $110^{\circ} \mathrm{C}$ which reveals an Arrhenius plot in a polymer matrix system but somewhat different from that of the Vogel-TammanFulcher (VTF) equation which can be described as follows.

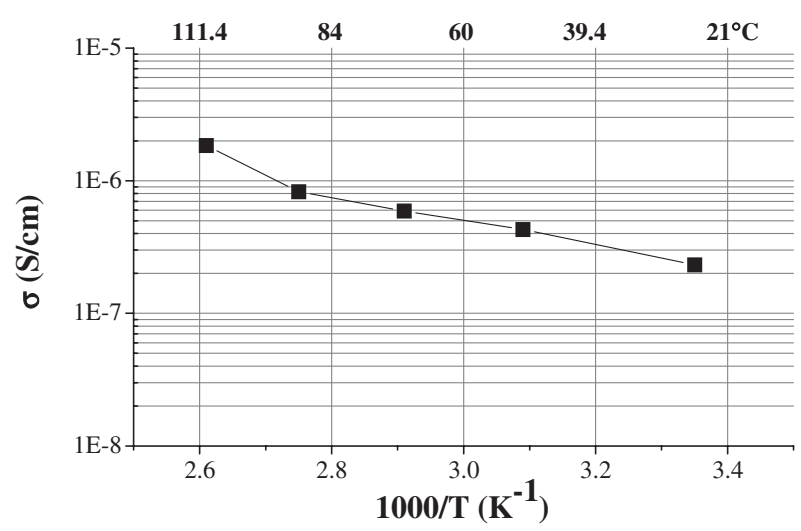

Figure 6. Temperature dependence on ionic conductivity of the SPE23 having 23:1 molar ratio of [EO]:[Li]. 


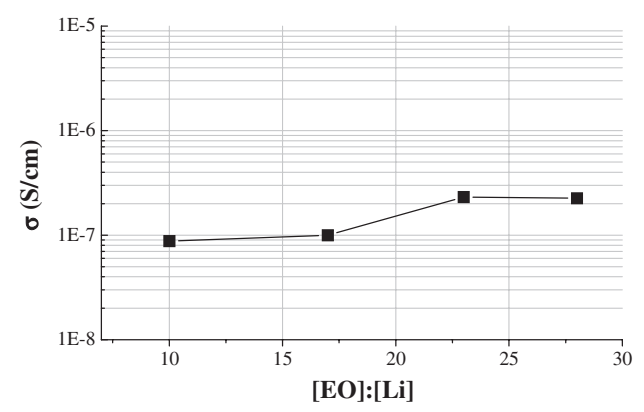

Figure 7. Ionic conductivity of single-ion polymer electrolytes as a function of $[\mathrm{EO}]:[\mathrm{Li}]$ ratio at room temperature.

$$
\sigma=A T^{-1 / 2} \exp \left[-\mathrm{E}_{\mathrm{a}} / k\left(T-T_{0}\right)\right]
$$

where $\sigma$ is the ionic conductivity, $A$ is proportional to the number of charge carriers in the system, $k$ is the Boltzmann constant, $T$ is a measured temperature and $T_{0}$ is a glass transition temperature $\left(T_{\mathrm{g}}\right)$ of polymer matrix. This result may be explained by a different conduction behavior of the lithium ion, caused by formation of a channel-like structure due to the hydrophobic and hydrophilic selfassembly. In such system, the lithium ion conduction behavior is deviated from the amorphous counter part. ${ }^{24}$ At this point, however, there was no strong evidence of the decoupled lithium ion motions, possibly due to the low ethylene oxide content. Figure 7 shows a [EO]:[Li] dependence on the ionic conductivity at room temperature. The ionic conductivity seems to be increased with decreasing ion content although the scale is small. This result can be ascribed to the formation of soft polymer matrix with lower content of stiff lithium methacrylate, which is unfavorable for lithium ion conduction especially at low temperature. As a result, the maximum room temperature ionic conductivity was obtained from the polymer sample having [EO]:[Li] ratio of 23:1 or $28: 1$.

The single-ion conduction behavior can be demonstrated by a potentiostatic polarization test (or steady-state current method); a constant potential is applied to a lithium symmetric cell, which is non-blocking to lithium ion but blocking to counter anion and the current is observed. In our study, a step signal was applied to a lithium symmetric cell for a certain period of time and the current response under that condition was observed as a function of time at room temperature. The current respond completely in phase to stepped potential and the result indicates that there was no active migration of counter anion thus no polarizations occur as shown in Figure 8. Comparing with salt-doped polymer electrolyte system which generally shows a significant polarization in a time frame of few second, ${ }^{25}$ it is worth to notice that tethered counter anion to the polymer backbone is useful method to prevent undesired polarization thus guarantee a high lithium ion transference number.

Conclusively, we have shown how the lithium ion concentration effects on the domain size of crystalline phase to combine lithium ion conduction and crystalline domains into an inner-outer double cylinder like array through a less studied macromonomer structure. As a result, the more the lithium ion concentration, the lower the melting temperature of crystalline phase was observed due to the enhanced coordination between lithium and ethylene oxide unit, thereby shrinking of heptadecane domains. In other words, it seems difficult to find such interesting result from a common polymer electrolytes consisted with PEO methacrylate, poly(heptadecane methacrylate) and poly(lithium methacrylate) separately. Therefore, it is worth to utilize a designed macromonomer in polymer electrolyte synthesis to elucidate the structure and property relationship. The self-assembled structure was also supported by the ion conduction behavior of polymer electrolytes which is deviated from the VTF equation. Although the polymer electrolytes exhibited low ionic conductivity due to the low ethylene oxide content, it is obvious that the system has a crystalline conduction behavior through the PEO channel and a single-ion conduction nature.

Acknowledgment. This work was conducted in the laboratories of Prof. Anne Mayes and Prof. Yang Shao-Horn at the Massachusetts Institute of Technology.

Received: January 28, 2008

Accepted: April 18, 2008

Published: July 2, 2008
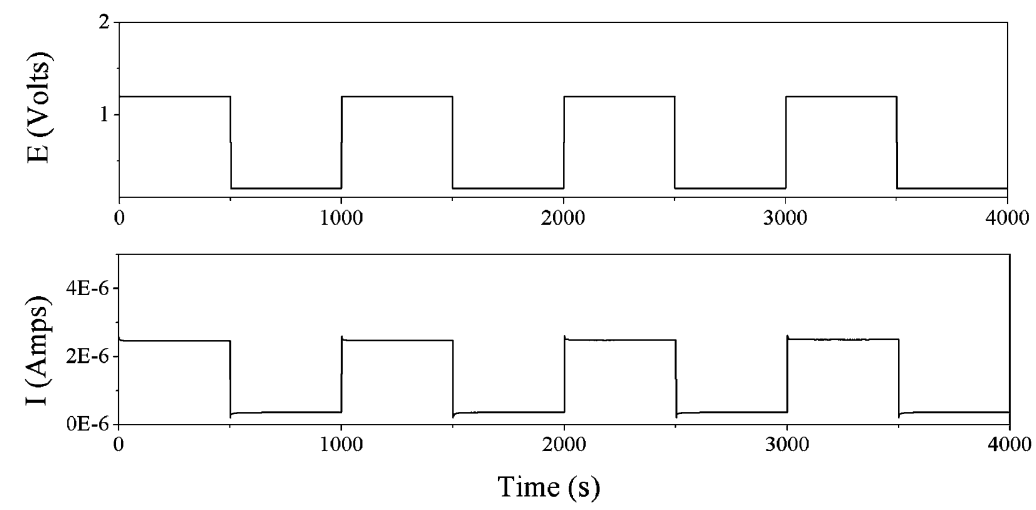

Figure 8. DC-polarization test at room temperature for SPE in a lithium symmetric cell. 


\section{REFERENCES}

1. D. E. Fenton, J. M. Parker, and P. V. Wright, Polymer, 14, 589 (1973).

2. B. Scrosati and C. A. Vincent, MRS Bulletin, 25, 28 (2000).

3. P. Arora, M. Doyle, A. S. Gozdz, R. E. White, and J. Newman, J. Power Source, 88, 219 (2000).

4. H. L. Dai and T. A. Zawodzinski, J. Electrochem. Soc., 143, L107 (1996).

5. W. A. van Schalkwijk and B. Scrosati, "Advances in Lithium-Ion Batteries," Kluwer Academic Publishers, New York, 2002.

6. "Lithium Batteries Science and Technology," G. A. Nazri and G. Pistoia, Ed., Kluwer Academic Publishers, New York, 2004.

7. M. Doyle, T. F. Fuller, and J. Newman, Electrochim. Acta, 39, 2073 (1994).

8. I. Rey, J. C. Lassegues, P. Baudry, and H. Majastre, Electrochim. Acta, 43, 1539 (1998).

9. Y. Ma, M. Doyle, T. F. Fuller, M. M. Doeff, L. C. De Jonghe, and J. Newman, J. Electrochem. Soc., 142, 1859 (1995).

10. E. Tsuchida, H. Ohno, and N. Kobayashi, Macromolecules, 21, 96 (1988).

11. T. Fujinami, A. Tokimun, M. A. Mehta, D. F. Shriver, and G. C. Rawsky, Chem. Mat., 9, 2236 (1997).

12. K. Ito, Y. Tominaga, and H. Ohno, Electrochim. Acta, 42, 1561 (1997).

13. J. M. G. Cowie and G. H. Spence, Solid State Ionics, 123, 233 (1999).
14. S. S. Zhang, Z. Chang, K. Xu, and C. A. Angell, Electrochim. Acta, 45, 1229 (2000).

15. Z. Florjanczyk, W. Bzducha, N. Langwald, J. R. Dygas, F. Krok, and B. Misztal-Faraj, Electrochim. Acta, 45, 3563 (2000).

16. X. G. Sun, W. Xu, S. S. Zhang, and C. A. Angell, J. Phys.: Condens. Matter, 13, 8235 (2001).

17. D. R. Sadoway, B. Huang, P. E. Trapa, P. P. Soo, P. Bannerjee, and A. M. Mayes, J. Power Sources, 97-98, 621 (2001).

18. N. Kobayashi, M. Uchiyama, and E. Tsuchida, Solid State Ionics, 17, 307 (1985).

19. D. Benrabah, S. Sylla, F. Alloin, G. C. Sanchez, and M. Armand, Electrochim. Acta, 40, 2259 (1995).

20. S.-W. Ryu, P. E. Trapa, S. C. Olugebefola, J. A. Gonzalez-Leon, D. R. Sadoway, and A. M. Mayes, J. Electrochem. Soc., 152, A158 (2005).

21. F. Chia, Y. Zheng, J. Liu, N. Reeves, G. Ungar, and P. V. Wright, Electrochim. Acta, 48, 1939 (2003).

22. Y. Zheng, F. Chia, G. Ungar, and P. V. Wright, J. Power Sources, 97-98, 641 (2001)

23. Y. Zheng, F. Chia, G. Ungar, and P. V. Wright, Electrochim. Acta, 46, 1397 (2001).

24. Z. Gadjourova, Y. G. Andreev, D. P. Tunstall, and P. C. Bruce, Nature, 412, 520 (2001).

25. V. Mauro, A. D'Aprano, F. Croce, and M. Salomon, J. Power Sources, 141, 167 (2005). 